\title{
Présentation efficace des données dans les tableaux et les figures
}

\author{
Patricia Huston ${ }^{1}$
}

\section{Résumé}

La présentation des données dans les tableaux et les figures est une marque distinctive des publications scientifiques. Les tableaux et les figures gagnent en efficacité lorsqu'ils reflètent deux principes et un certain nombre de pratiques exemplaires. Le premier principe consiste à utiliser des tableaux et des figures pour mettre en évidence les principales constatations d'une étude. Le deuxième principe consiste à choisir le format approprié en fonction du type de données. Les tableaux sont particulièrement efficaces pour présenter des données précises et des résultats multiples. Les figures sont plus efficaces pour présenter les tendances au fil du temps ou les valeurs comparatives. Lors de la conception d'un tableau, différents groupes sont précisés dans des colonnes, puis comparés en fonction de variables présentées dans les rangées. Cette structure permet des comparaisons entre les différents groupes étudiés. Lors de la conception de figures, la variable indépendante (comme le temps) se trouve sur l'axe des abscisses $(x)$, et la variable dépendante ou la variable de résultat se trouve sur l'axe des ordonnées $(y)$. De bons titres pour les tableaux et les figures donnent une description concise de qui, quoi, où, quand et combien. Les lecteurs électroniques peuvent lire les tableaux si les rangées et les colonnes sont séparées au moyen de lignes visibles et s'il y a une seule donnée par cellule; les lecteurs électroniques peuvent lire les figures s'il y a un lien vers une feuille de calcul Excel contenant les données ou s'il y a une brève description textuelle. Grâce à ces principes et pratiques exemplaires, les tableaux et les figures mettront en lumière les principales conclusions des études scientifiques d'une manière claire, accessible et mémorable.
Cette oeuvre est mise à la disposition selon les termes de la licence internationale Creative Commons Attribution 4.0

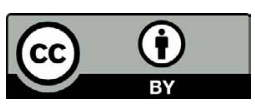

Affiliation

${ }^{1}$ Bureau du conseiller scientifique principal, Agence de la santé publique du Canada, Ottawa, ON (au moment de la rédaction de l'article)

${ }^{\star}$ Correspondance :

phac.ccdr-rmtc.aspc@canada.ca

Citation proposée : Huston P. Présentation efficace des données dans les tableaux et les figures. Relevé des maladies transmissibles au Canada 2020;46(7/8):271-6. https://doi.org/10.14745/ccdr.v46i78a05f

Mots-clés : tableaux, figures, publications scientifiques, présentation de données, pratiques exemplaires

\section{Introduction}

La présentation des données est une marque distinctive d'une étude scientifique; pourtant, les auteurs ne tiennent souvent pas compte de la façon dont ils vont présenter leurs données avant de commencer à préparer des manuscrits pour publication. Des ouvrages de référence sur ce sujet sont disponibles (1-4), tout comme des articles dans d'autres disciplines (5-7). Cependant, ces sources ne tiennent généralement pas compte des lignes directrices internationales pour s'assurer que les publications en ligne sont accessibles aux personnes atteintes d'une déficience visuelle (8). Ces lignes directrices ont été adoptées par les éditeurs scientifiques du monde entier, et savoir comment s'y conformer évite la nécessité d'une révision ultérieure.

Le présent article vise à fournir aux chercheurs un aperçu concis des principes et des pratiques exemplaires pour la création de tableaux et de graphiques accessibles dans le domaine de la recherche en santé. Des renseignements sur d'autres types de figures, comme les photographies, les diagrammes et les images biomédicales, se trouvent ailleurs (1).

\section{Utilisation appropriée}

Les tableaux et les figures sont souvent essentiels pour communiquer la recherche en santé. Pour présenter efficacement les données, vous devez appliquer deux principes clés et certaines pratiques exemplaires. Les deux principes clés sont de mettre en évidence les principales constatations et de choisir le format approprié en fonction du type de données. Les pratiques exemplaires comprennent les conventions de création de titres; le découpage des tableaux et des figures; et la présentation accessible des abréviations et des notes en bas de page pour qu'ils puissent être lus par ceux qui utilisent les lecteurs électroniques avec la technologie de synthèse vocale (9).

La question de la quantité de données à inclure se pose souvent au moment de la préparation des résultats de recherche pour publication. Le réflexe initial pourrait être de partager tout ce qui a été recueilli. Avec la tendance croissante vers la science ouverte et les données ouvertes, cela est maintenant possible : des ensembles de données complets sont affichés et rendus accessibles en ligne (10). Toutefois, dans une publication 
scientifique, une orientation claire aide le lecteur à cerner et à conserver les principales conclusions d'une étude; trop de données peuvent être écrasantes. D'un autre côté, si les lecteurs reçoivent trop peu de données, ils peuvent avoir l'impression qu'il y a un manque de substance pour appuyer les conclusions scientifiques.

La meilleure façon de réussir à bien présenter les données dans les tableaux et les figures est d'utiliser ces données pour mettre l'accent sur les principales constatations, y compris les résultats de la méthodologie (expliqué plus en détail ci-après) et les principales mesures des résultats de l'étude. Sachez que, dans le cadre de l'évaluation de la pertinence d'un manuscrit pour une revue, les rédacteurs évalueront si l'information fournie par les figures et les tableaux est justifiée en fonction de la longueur du document et si le manuscrit respecte les limites d'espace de la revue (11). En règle générale, les articles de revues médicales contiennent trois à quatre tableaux et figures, et de cinq à sept tout au plus.

\section{Méthodes}

Il existe au moins trois cas où un tableau ou une figure montrant des renseignements pertinents sur la méthodologie est indiqué : les essais cliniques randomisés, les examens systématiques et les études impliquant au moins deux groupes. Pour les essais cliniques randomisés, l'énoncé CONSORT a déterminé la nécessité d'un organigramme pour montrer le nombre de personnes invitées à participer à l'étude, combien ont acceptés et ont été randomisés, le nombre dans chaque groupe et le nombre ayant abandonné ou complété l'étude (12).

Dans les examens systématiques, l'énoncé PRISMA a mis en évidence la nécessité d'un organigramme pour montrer les résultats de la recherche documentaire et la présélection des études en fonction des critères d'inclusion et d'exclusion (13).

Dans les études épidémiologiques impliquant deux groupes ou plus (comme une étude cas-témoin imbriquée), l'énoncé STROBE souligne la nécessité de décrire les caractéristiques démographiques, cliniques et sociales des participants à l'étude, ainsi que l'information sur les expositions et les facteurs de confusion possibles pour les cas et les contrôles (14); un tableau est la façon la plus efficace de le faire. Un tableau aide également les lecteurs à déterminer si les groupes étaient semblables au début de l'étude; la randomisation ne donne pas toujours lieu à des groupes équivalents. Dans les essais contrôlés randomisés et les études de cas, si les membres d'un groupe présentent davantage de comorbidités, par exemple, cela peut biaiser les résultats et doit être pris en compte lors de I'interprétation des résultats.

\section{Principaux résultats}

Les principales conclusions de la recherche comprennent la principale mesure des résultats et, souvent, un certain nombre de mesures secondaires qui sont toutes liées à l'objectif de l'étude. L'objectif peut comporter plusieurs composantes. À titre d'exemple, si l'objectif d'une étude est d'examiner les tendances dans les nouveaux cas de $\mathrm{VIH}$ sur une certaine période en fonction de l'âge, du sexe, de l'emplacement géographique et du groupe de risque, chacun de ces domaines serait présenté dans les résultats. On s'attend à ce que les données de chacun de ces domaines soient présentées visuellement.

Une fois que vous avez déterminé ce que vous voulez mettre en évidence, la question est de savoir s'il est préférable de présenter les données dans un tableau ou une figure. La réponse dépend du type de données que vous devez présenter. Un graphique est préférable pour présenter une tendance au fil du temps. Si les valeurs exactes sont importantes, ou si elles sont nombreuses, un tableau est préférable (1).

\section{Figures}

Les figures peuvent fournir en un coup d'œil des renseignements dont l'explication nécessiterait autrement plusieurs phrases. Un graphique ou un histogramme montre facilement et mémorablement des données comparatives ou présente les tendances au fil du temps. À titre d'exemple, un graphique est couramment utilisé dans les résumés de surveillance et les rapports d'épidémie pour montrer l'incidence de la maladie.

Pour les graphiques, l'axe des y indique les mesures d'intérêt (comme les taux ou le nombre de cas) et commence généralement à zéro. L'axe des $x$ représente la variable indépendante, par exemple le temps (par jours, mois ou années) (voir la figure 1). Un tel affichage visuel permet de déterminer instantanément si les taux augmentent, diminuent ou restent à peu près les mêmes. Si vous devez condenser la hauteur du graphique, l'axe des y peut être une échelle logarithmique ou vous pouvez utiliser une paire de lignes diagonales (-- // --) pour montrer que l'échelle n'est pas continue.

La couleur peut être utilisée pour différencier les différentes lignes dans les graphiques ou les barres dans les histogrammes, mais pour respecter les directives d'accessibilité, les couleurs doivent être suffisamment différentes pour passer un test colorimétrique ou la présentation doit comporter une caractéristique de conception supplémentaire pour les distinguer. Les figures doivent être marquées d'une légende pour définir chaque ligne ou chaque barre. Dans la figure 1, la légende définit les données représentées par les barres rouges et les barres bleues; ces deux couleurs ont réussi les tests colorimétriques. 
Figure 1 : Envois de Corynebacterium diphtheriae pour des analyses de toxines par année et sous-ensemble en fonction du nombre de souches toxigènes, 2006 à 2019

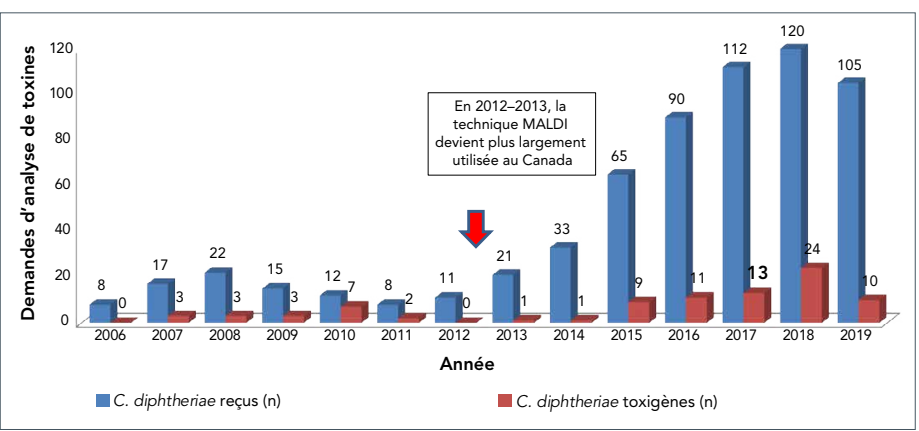

Abréviations : C. diphtheriae, Corynebacterium diphtheriae; MALDI, désorption-ionisation par A

Source : Bernard et al., 2019 (15)

\section{Tableaux}

Les résultats précis et les multiples types de données sont mieux présentés dans les tableaux. Lors de la conception de tableaux, il est utile de tenir compte à la fois de la structure et du placement du contenu.

\section{Structure}

Les auteurs se demandent souvent quelles données noter dans les rangées et quelles données noter dans les colonnes. Un principe général veut que les variables dépendantes ou les variables de résultat soient présentées dans les rangées et que les variables indépendantes soient présentées dans les colonnes (1). À titre d'exemple, si vous comparez les caractéristiques de deux groupes étudiés, les groupes étudiés seraient présentés dans les colonnes et les différentes caractéristiques ou résultats de base seraient présentés dans les rangées. La lecture de l'anglais et du français se fait horizontalement et l'indication des caractéristiques dans les colonnes permet aux lecteurs de comparer plus facilement les deux groupes.

Chaque colonne doit comprendre un en-tête qui décrit ce qui se trouve dans la colonne. Les intitulés qui couvrent deux colonnes ou plus sont appelés titre-accolade. À titre d'exemple, les titres-accolades peuvent décrire différents groupes (e.g. "Vaccination à deux doses documentée», "Vaccination à une dose documentée» et "Aucune documentation ou aucun historique de vaccination »). Sous chaque titre-accolade, il y a deux intitulés, soit «n» et «\%» (tableau 1 ).

L'intitulé de la colonne la plus à gauche décrit ce qui se trouve dans les rangées. À l'extrême gauche de chaque ligne se trouvent les rubriques. Les vedettes de la colonne de titres, qui organisent les en-têtes de ligne en groupes, peuvent être incluses. Chacune de ces vedettes décrit les données des cellules à droite. Considérons l'ordre des rubriques. Elles peuvent être présentées en ordre chronologique (e.g. "statut à l'admission", «statut au congé », «statut six mois après le congé »); par
Table 1: Noms et composantes d'un tableau scientifique ${ }^{a}$

\begin{tabular}{|c|c|c|c|c|c|c|c|}
\hline \multirow{2}{*}{\multicolumn{2}{|c|}{$\begin{array}{l}\text { En-tête de colonne } \\
\text { pour les titres- } \\
\text { accolades et les } \\
\text { vedettes de la } \\
\text { colonne de titres }\end{array}$}} & \multicolumn{2}{|c|}{$\begin{array}{c}\text { Titre- } \\
\text { accolade }\end{array}$} & \multicolumn{2}{|c|}{$\begin{array}{c}\text { Titre- } \\
\text { accolade }\end{array}$} & \multicolumn{2}{|c|}{ Total } \\
\hline & & $n$ & $\%$ & $n$ & $\%$ & $n$ & $\%$ \\
\hline \multirow{3}{*}{$\begin{array}{l}\text { Vedette } \\
\text { de la } \\
\text { colonne } \\
\text { de titres }\end{array}$} & Rubrique 1 & - & - & - & - & - & - \\
\hline & Rubrique 2 & - & - & - & - & - & - \\
\hline & Rubrique (n) & - & - & - & - & - & - \\
\hline \multirow{3}{*}{$\begin{array}{l}\text { Vedette } \\
\text { de la } \\
\text { colonne } \\
\text { de titres }\end{array}$} & Rubrique 1 & - & - & - & - & - & - \\
\hline & Rubrique 2 & - & - & - & - & - & - \\
\hline & Rubrique (n) & - & - & - & - & - & - \\
\hline Total & - & - & - & - & - & - & 100 \\
\hline
\end{tabular}

ordre alphabétique (comme les noms de différents pays si vous comparez leur incidence de maladies) ou géographiquement (e.g. incidence de maladies au Canada par province ou territoire de l'ouest à l'est). Assurez-vous que chaque titre-accolade, intitulé, vedette de la colonne de titre et rubrique décrit avec précision ce qui est présenté dans les cellules qu'il englobe.

Écrivez seulement une donnée par cellule et ne laissez aucune cellule vide. La convention consistait à écrire la valeur "n», suivie du pourcentage entre crochets dans une seule cellule. Comme le montre le tableau 1, ces données sont maintenant écrites dans des colonnes distinctes afin d'être interprétées avec précision par les lecteurs électroniques $(8,9)$. Si la référence est zéro, écrivez zéro. S'il n'y a pas de données, écrivez « $\mathrm{SO}$ » (pour «sans objet») et expliquez l'abréviation sous le tableau.

\section{Contenu}

Habituellement, les résultats numériques dans les tableaux sont justifiés à droite et les nombres sont alignés sur le point décimal (1). Si la taille totale de l'échantillon est inférieure à 100, représentez les pourcentages par des nombres entiers (i.e. pas de décimales) pour ne pas donner l'impression d'une plus grande précision que ce qui est approprié (3). De même, si la taille totale de l'échantillon est inférieure à 20 , aucun pourcentage n'est nécessaire (3). Si les unités varient dans une colonne (e.g. si vous rendez compte de différents résultats d'analyse de sang), les unités doivent être présentées dans la rubrique et les données de chaque cellule peuvent être centrées.

Au besoin, ajouter une colonne pour une mesure statistique de variation, comme l'écart type ou l'erreur type de la moyenne, et une autre colonne pour la valeur $p$ (11).

En général, l'échantillon complet de l'étude doit être pris en compte (3). Si vous ne possédez pas de données pour certains éléments (e.g. si un participant à l'enquête n'a pas répondu à certaines questions), envisagez d'ajouter une catégorie «pas de réponse » afin que les lecteurs puissent considérer l'effet de 
cette réponse sur les résultats globaux. En rapport avec cette pratique, le nombre réel (ou « $n$ ») et le pourcentage devraient être présentés, et tous les pourcentages devraient totaliser $100 \%$ (3). Pour le démontrer, il y a souvent un total dans la colonne extrême de droite et/ou dans la rangée inférieure.

Une fois la table construite, il y a quelques touches de finition à considérer, par exemple, la manière de réduire au minimum l'espace vide. Pour les tableaux textuels, utilisez des abréviations et des symboles pour réduire la largeur des colonnes, puis ajustez les largeurs de colonnes de sorte que les colonnes qui contiennent le plus de renseignements aient la plus grande largeur. Enfin, comparez toujours les chiffres du tableau aux données d'origine et assurez-vous que toutes corrections soient reflétées dans le texte.

\section{Pratiques exemplaires}

Il existe un certain nombre de pratiques exemplaires qui couvrent tous les autres renseignements qui peuvent être associés aux tableaux et aux figures. Le présent résumé est fondé sur le manuel de style formel pour les publications scientifiques, Scientific Style and Format (1), les Recommendations for the Conduct, Reporting, Editing and Publication of Scholarly Work in Medical Journals internationales (15) et les Web Content Accessibility Guidelines (8).

\section{Titres}

Le titre d'un tableau ou d'une figure doit fournir suffisamment de renseignements pour expliquer les données du tableau sans qu'il soit nécessaire de lire le contexte dans l'article. Considérez chaque tableau et chaque figure comme étant "autonome». Le titre doit être concis, mais complet, et inclure qui et quoi, quand et où et, au besoin, la taille totale de l'échantillon. Lorsqu'un ensemble de tableaux ou de figures contient des types de renseignements similaires, un format parallèle est utilisé pour les titres. À titre d'exemple :

- Figure 1 : Nouveaux cas de VIH au Canada, 2019, par région géographique $(n=)$

- Figure 2 : Nouveaux cas de VIH au Canada, 2019, selon l'âge et le sexe $(n=)$

- Figure 3 : Nouveaux cas de VIH au Canada, 2019, par facteur de risque $(n=)$

\section{Abréviations, notes en bas de page et références}

Les abréviations sont généralement présentées dans la ligne immédiatement sous un tableau ou une figure et sont énumérées par ordre alphabétique (e.g. AST, aspartate aminotransférase; CFA, conduite avec facultés affaiblies, etc.). Même si des abréviations ont été présentées dans le texte, elles devraient être redéfinies sous un tableau ou une figure pour que ces derniers puissent être autonomes.

Des renvois sont utilisés lorsque des explications supplémentaires sont nécessaires. Les notes de bas de page sont présentées en inscrivant une lettre en exposant (en commençant par «a», puis en suivant l'ordre alphabétique) immédiatement après les mots ou les chiffres qui nécessitent une explication supplémentaire. Les chiffres sont évités pour les notes de bas de page, puisque les lecteurs électroniques peuvent les confondre avec les résultats ou les numéros de référence. Les notes de bas de page sont présentées séquentiellement dans le même ordre qu'une personne lit-de gauche à droite dans les rangées et de haut en bas. Comme le montre le tableau 1, les notes de bas de page sont placées séquentiellement, sous la ligne des abréviations.

Les références sont indiquées dans les tableaux pour indiquer la source d'un tableau ou pour appuyer une assertion dans un renvoi. Les références citées uniquement dans les tableaux ou les légendes des figures doivent être numérotées en fonction de l'endroit où le tableau ou la figure est cité pour la première fois dans le texte. Par exemple, la figure 1 ci-dessus contient une référence après le titre qui maintient les citations en ordre numérique.

\section{Données supplémentaires}

Si vous utilisez des données provenant d'autres sources publiées ou non publiées qui ne sont pas du domaine public, vous devez soumettre à votre éditeur une autorisation écrite du titulaire du droit d'auteur pour reproduire ces données (11). La tendance actuelle est à l'abandon des articles protégés par le droit $d^{\prime}$ auteur en faveur du domaine public, comme avec une licence Creative Commons (16). Dans tous les cas, il est important de citer la source et d'indiquer si des changements ont été apportés à l'original.

Parfois, des tableaux supplémentaires contenant des données de sauvegarde peuvent être appropriés dans une annexe ou un supplément ou ils sont mis à la disposition des lecteurs directement par les auteurs sur demande. C'est quelque chose qui est négocié avec le rédacteur de la publication. Lorsqu'une entente est conclue à cet égard, une déclaration est ajoutée au texte pour informer les lecteurs que ces renseignements supplémentaires sont disponibles et les informer de l'endroit où ils se trouvent. Des données supplémentaires sont généralement incluses dans le manuscrit au moment de la soumission, de sorte que les données soient disponibles dans le cadre du processus d'examen par les pairs.

\section{Mention dans le texte}

Chaque tableau et figure d'un article doit être présenté dans le texte. Les tableaux et les figures sont numérotés en ordre et, selon la convention de publication, ils sont placés à la fin du 
Tableau 2 : Description textuelle de la figure 1 pour les personnes ayant une déficience visuelle

\begin{tabular}{|c|c|c|c|c|c|c|c|c|c|c|c|c|c|c|c|}
\hline \multirow{2}{*}{ Espèces } & \multicolumn{15}{|c|}{ Souches toxigènes/année } \\
\hline & 2006 & 2007 & 2008 & 2009 & 2010 & 2011 & 2012 & 2013 & 2014 & 2015 & 2016 & 2017 & 2018 & 2019 & Total \\
\hline $\begin{array}{l}\text { C. ulcerans } \\
\text { reçus (n) }\end{array}$ & 0 & 3 & 1 & 0 & 0 & 0 & 0 & 4 & 5 & 1 & 0 & 1 & 2 & 5 & 22 \\
\hline $\begin{array}{l}\text { C. ulcerans } \\
\text { toxigènes (n) }\end{array}$ & 0 & 1 & 1 & 0 & 0 & 0 & 0 & 1 & 4 & 0 & 0 & 0 & 1 & 2 & 10 \\
\hline
\end{tabular}

paragraphe où ils sont présentés pour la première fois. Toutefois, pour la soumission du manuscrit, la plupart des revues médicales demandent que les auteurs placent les tableaux et les figures à la fin du manuscrit (11). Cela permet aux éditeurs et aux réviseurs de se concentrer séparément sur le texte et la présentation des données. Les tableaux et les figures sont placés dans le texte pendant la mise en page conformément à la convention, mais cela peut être légèrement modifié pour assurer une présentation agréable de l'article. Il est utile de connaître cette convention et d'éviter de faire des déclarations comme : "Voir les tableaux 1 à $4 »$, ce qui signifie que le texte serait normalement suivi de quatre tableaux, ce qui divise le flux du texte et crée des difficultés de formatage sur la page imprimée.

\section{Accessibilité}

Les lecteurs électroniques sont en mesure de lire les tableaux - tant qu'il n'y a qu'une seule donnée dans chaque cellule - mais ils ne peuvent pas lire les figures. Pour contourner le problème, il faut inclure une feuille de calcul Excel (pour les graphiques, les histogrammes et les graphiques circulaires) ou ajouter une description textuelle (pour les organigrammes et les illustrations). Le tableau 2 montre un tableau de type Excel utilisé pour présenter les données de la figure 1. Dans la version HTML de la publication originale, un lien hypertexte vers le tableau se trouvait à l'expression "Description textuelle» qui se trouve sous la figure (15).

\section{Conclusion}

La création de tableaux et de chiffres efficaces est essentielle pour communiquer avec succès la recherche scientifique. Lorsqu'ils sont conçus pour mettre en lumière les principales conclusions d'une étude et qu'ils sont fondés sur les pratiques exemplaires, les tableaux et les figures aident à présenter les résultats d'une étude scientifique de manière claire, accessible et mémorable.

\section{Déclaration de l'auteur}

P. H. a conçu et rédigé l'article.

\section{Intérêts concurrents}

Aucun.

\section{Références}

1. Style Manual Subcommittee, Council of Science Editors. Scientific Style and Format: The CSE Manual for Authors, Editors, and Publishers. 8th ed. Wheat Ridge (CO): Council of Science Editors; 2014.

2. Day RA, Gastel B. How to write and publish a scientific paper. 7th ed. Cambridge (UK): Cambridge University Press; 2017.

3. Lang TA, Secic M. How to report statistics in medicine: annotated guidelines for authors, editors, and reviewers. 2nd ed. Philadelphia (PA): American College of Physicians; 2006.

4. Moher D, Altman D, Schulz K, Simera I, Wager E, editors. Guidelines for reporting health research: a user's manual. Hoboken (NJ): John Wiley \& Sons; 2014.

5. Kotz D, Cals JW. Effective writing and publishing scientific papers, part VII: tables and figures. J Clin Epidemiol 2013;66(11):1197. DOI PubMed

6. Bahadoran Z, Mirmiran P, Zadeh-Vakili A, Hosseinpanah F, Ghasemi A. The principles of biomedical scientific writing: results. Int J Endocrinol Metab 2019;17(2):e92113. DOI PubMed

7. Boers M. Graphics and statistics for cardiology: designing effective tables for presentation and publication. Heart 2018;104(3):192-200. DOl PubMed

8. W3C. Web content accessibility guidelines (WCAG) 2.1. Cambridge (MA): World Wide Web Consortium; 2018 Jun 5 (accédé 2020-04-25). https://www.w3.org/TR/WCAG21/

9. The Understood Team. Text-to-speech technology: what it is and how it works. Understood (accédé 2020-0325). https://www.understood.org/en/school-learning/ assistive-technology/assistive-technologies-basics/text-t o-speech-technology-what-it-is-and-how-it-works

10. Huston P, Edge VL, Bernier E. Tirer profit des données ouvertes en santé publique. Relevé des maladies transmissibles au Canada 2019;45(11):277-82. DOI

11. International Committee of Medical Journal Editors. Recommendations for the conduct, reporting, editing and publication of scholarly work in medical journals. ICMJE (accédé 2020-03-25). www.ICMJE.org 
12. Schulz KF, Altman DG, Moher D; CONSORT Group. CONSORT 2010 Statement: updated guidelines for reporting parallel group randomised trials. Trials 2010;11:32. DOI PubMed

13. Liberati A, Altman DG, Tetzlaff J, Mulrow C, Gøtzsche PC, loannidis JP, Clarke M, Devereaux PJ, Kleijnen J, Moher D. The PRISMA statement for reporting systematic reviews and meta-analyses of studies that evaluate healthcare interventions: explanation and elaboration. BMJ 2009;339:b2700. DOI PubMed

14. von Elm E, Altman DG, Egger M, Pocock SJ, Gøtzsche PC, Vandenbroucke JP; STROBE Initiative. The Strengthening the Reporting of Observational Studies in Epidemiology (STROBE) statement: guidelines for reporting observational studies. J Clin Epidemiol 2008;61(4):344-9.

DOI PubMed
15. Bernard KA, Pacheco AL, Burdz T, Wiebe D. Increase in detection of Corynebacterium diphtheriae in Canada: 2006-2019. Can Commun Dis Rep 2019;45(11):296-301. DOI PubMed

16. Creative Commons. Attribution 4.0 International (CC BY 4.0) (accédé 2020-03-25). https://creativecommons.org/licenses/ by/4.0/deed.fr

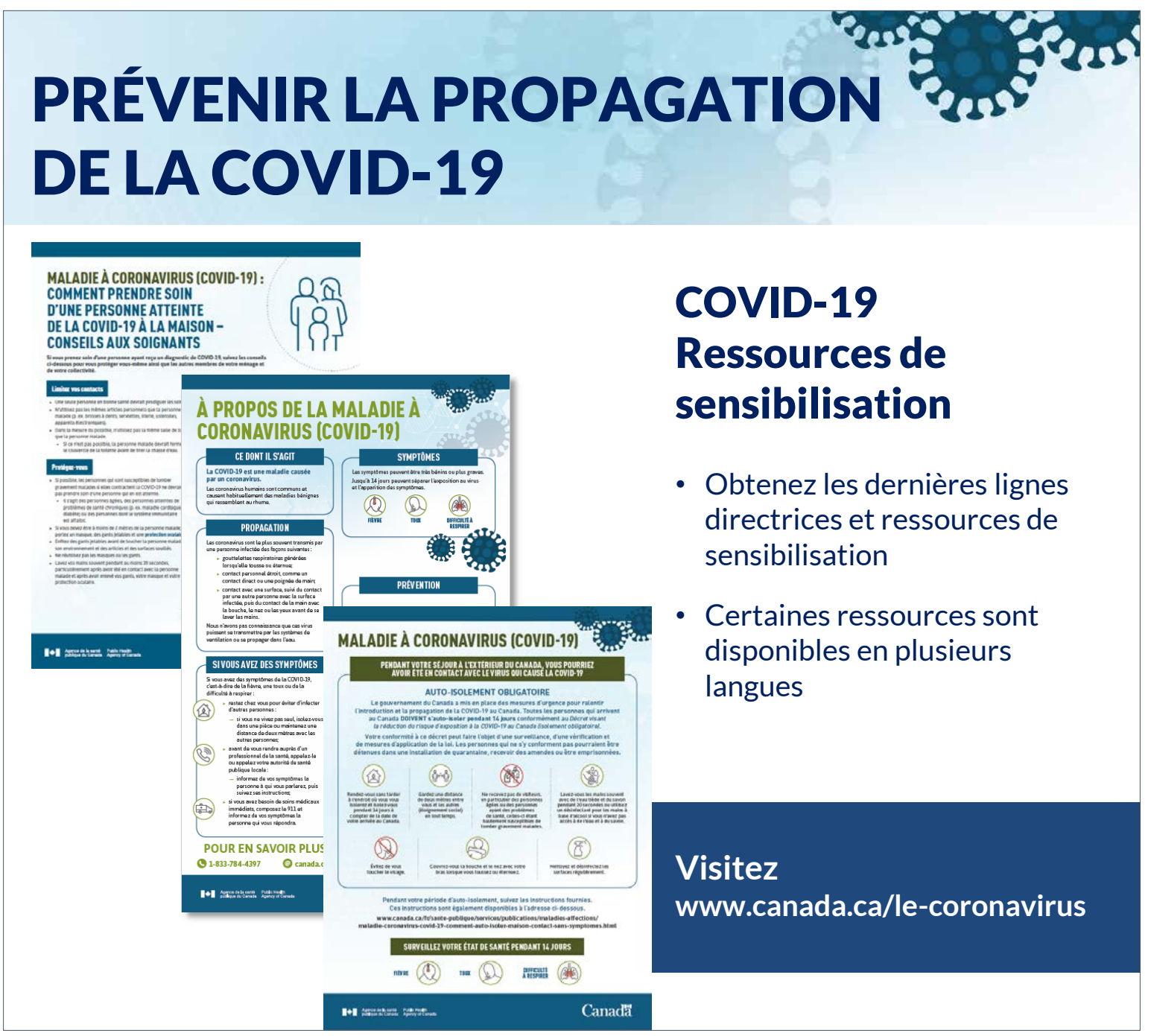

